\title{
A new mechanism for the silica nanoparticle dispersion-agglomeration transition in a poly(methyl methacrylate)/silica hybrid suspension
}

\author{
Tsuyoshi Tadano ${ }^{1}$, Rui Zhu ${ }^{2}$, Yoshio Muroga ${ }^{3}$, Toru Hoshi ${ }^{3}$, Daisuke Sasaki ${ }^{3}$, Shoichiro Yano ${ }^{3}$ \\ and Takashi Sawaguchi ${ }^{3}$
}

We examined the agglomeration behavior of a suspension of $\mathrm{SiO}_{2}$ nanoparticles with average dimensions of $\sim 15 \mathrm{~nm}$ in a solution of tetrahydrofuran and polydisperse poly(methyl methacrylate) (PMMA) with a weight-average molecular weight in the range of (0.3-31) $\times 10^{4}$. For PMMA with a critical molecular weight $\left(M_{\mathrm{c}}\right)$ of $\sim 3 \times 10^{4}$ or larger, at which PMMA chains show effective entanglement, a critical polymer concentration $\left(C^{*}\right)$ was clearly observed. At $C^{*}$, the dispersed $\mathrm{SiO}_{2}$ nanoparticles came into contact with one another and rapidly agglomerated. $C^{*}$ increased with decreasing molecular weight. However, no clear $C^{*}$ was observed for PMMA $\left(M_{\mathrm{w}}: 0.3 \times 10^{4}\right)$ with $M_{\mathrm{c}}$ or lower molecular weights. The molecular weight dependence of the observed $C^{*}$ can be explained by the depletion effect, but the lack of a clear $C^{*}$ for low molecular weight PMMA cannot be explained with this theory. Because $C^{*}$ occurs in the vicinity of the critical concentration, at which the random coils in the solution come into contact with one another and begin to overlap, the entanglement of random coils is considered to be the driving force behind nanoparticle agglomeration. However, no $C^{*}$ was observed because effective entanglement does not occur for PMMA with $M_{\mathrm{c}}$ or lower.

Polymer Journal (2014) 46, 342-348; doi:10.1038/pj.2014.6; published online 26 March 2014

Keywords: depletion aggregation; dispersion-agglomeration transition; entanglement agglomeration; molecular weight dependence; poly(methyl methacrylate); silica nanoparticle; suspension

\section{INTRODUCTION}

Polymer composites are expected to form with nanoparticles uniformly dispersed in the polymer matrix. ${ }^{1-5}$ When the distances between the nanoparticles are on the sub-nanometer scale, however, an attractive force acts between the particles and aggregation occurs. The quantitative theory explaining the interaction between particles in a colloid dispersion is known as the DLVO theory. ${ }^{6-8}$ The total interaction energy $V_{p}$, expressed as the sum of the dispersion factors (electrostatic or steric repulsion) and of the aggregation factors (Van der Waals attraction), is determined by the distance between particles. When the distance between particles becomes extremely small, approaching $0.5 \mathrm{~nm}$, Born repulsion occurs, an energy minimum $V_{p \min }$ is obtained, and dense aggregation results. Because this aggregation is irreversible, redispersion is difficult to achieve. To stabilize particle dispersions, therefore, the ability to achieve an energy barrier (maximum value $V_{p \max }$ ) of approximately $15 k T$ (where $k$ is the Boltzmann constant and $T$ is the absolute temperature) at room temperature for a distance between particles of $0.5 \mathrm{~nm}$ or larger becomes important. In a system of colloidal particles dispersed in a solvent, the particles approach each other through Brownian motion, and interactions occur. More specifically, the dispersion can be sustained when there is repulsion due to the electrical double layer between the surfaces of the particles or when three-dimensional effects are present, such as solvation of the colloidal particle surfaces, adsorption of a surface active agent or chemical modification (grafting). Considerable effort has been made to uniformly disperse nanoparticles in a matrix because they are prone to agglomeration.

Blending in an organic solvent has been cited as an effective method for preparing nanocomposites. A recent successful example was reported by Meth and co-workers. ${ }^{9}$ To stabilize a uniform colloidal dispersion of nanosilica $\left(\mathrm{SiO}_{2}\right)$ particles with average dimensions of $28.6 \pm 0.3 \mathrm{~nm}$ in polystyrene or poly(methyl methacrylate) (PMMA), the particle surfaces were capped with phenyl groups using a sol-gel reaction between the silanol groups $(\mathrm{SiOH})$ on the particle surfaces and phenyltrimethyl methoxysilane. This phenyl-modified nanosilica could be dispersed uniformly at a relatively high concentrations in dimethyl formamide containing just a minute amount of water. After stirring in roll mill blends of organo-

${ }^{1}$ NIKKA SEIKO CO., Setagaya-ku, Tokyo, Japan; ${ }^{2}$ Department of Materials and Applied Chemistry, Graduate School of Science and Technology, Nihon University, Chiyoda-ku, Tokyo, Japan and ${ }^{3}$ Department of Materials and Applied Chemistry, College of Science and Technology, Nihon University, Chiyoda-ku, Tokyo, Japan

Correspondence: Professor T Sawaguchi, Department of Materials and Applied Chemistry, College of Science and Technology, Nihon Univesity, 1-8-14 Kandasurugadai, Chiyoda-ku, Tokyo 101-8308, Japan.

E-mail: sawaguchi.takashi@nihon-u.ac.jp

Received 12 October 2013; revised 15 January 2014; accepted 17 January 2014; published online 26 March 2014 
colloidal $\mathrm{SiO}_{2}$ with either two types of high molecular weight PMMA or two types of polystyrene, the suspension was cast onto a glass plate or onto a polyimide (Kapton) sheet, and the solvent was then removed under vacuum at $110^{\circ} \mathrm{C}$. The glass transition temperature was relatively constant because the thickness of the composite films was $5-20 \mu \mathrm{m}$ and because the specimen used for the nanosilica content had a high molecular weight, resulting in a stable uniform dispersion at up to 50 vol $\%$ nanosilica.

The dispersion was considered stable in organic solvent because of the increase in affinity between the particle surface and the polymer due to the surface modification of the colloidal $\mathrm{SiO}_{2}$. The agglomeration of colloidal particles dispersed in an organic solvent with polymers that exhibit only weak hydrogen bonding or similar interactions has long been discussed on the basis of the theory of depletion aggregation. ${ }^{10,11}$ The aggregation of colloidal particles is predicted to be related to the dimensions of the dissolved polymers, specifically the diameters of their random-coils, because the chains of polymers (random coils) caught between the colloidal particles or plates are believed to undergo elastic deformation when a driving force is applied to move them closer. This deformation clears the polymers from between the colloidal particles, resulting in interparticle contact. Applications have been developed based on this theory. ${ }^{12,13}$ In particular, Vrij and co-workers used a cyclohexane suspension of spherical $\mathrm{SiO}_{2}$ nanoparticles and monodisperse polystyrene with different molecular weights to evaluate the critical polymer concentration at which the $\mathrm{SiO}_{2}$ nanoparticles would agglomerate because of the depletion effect and also evaluated the molecular weight dependence of this critical concentration. ${ }^{14,15}$ They found that the critical concentration was often lower for higher molecular weights, or in other words, for larger diameter random coils, and that when the dimensions of the $\mathrm{SiO}_{2}$ nanoparticles and the initial concentration were increased, the depletion effect became more significant and thus the agglomeration became more evident. However, no clear experimental results have been reported, and there has been no discussion of the limitations of the polymer solution theory or of the depletion effects pertaining to the critical polymer concentration that triggers the depletion effect.

We evaluated the agglomeration behavior of organo- $\mathrm{SiO}_{2}$ nanoparticles randomly dispersed in a hybrid suspension of tetrahydrofuran (THF) and PMMA to determine the effect of the polymers on the nanoparticle dispersion-agglomeration transition behavior of the polymer/nanoparticle composites. In particular, the molecular weight dependence of the critical polymer concentration at which the nanosilica would agglomerate was determined, even for polydisperse PMMA. ${ }^{16,17}$ Moreover, the dispersion-agglomeration transition phenomenon was retained in the hybrid film using the hybrid suspension. ${ }^{18}$ In this study, the molecular weight dependence of the critical polymer concentration for a particular dispersion-agglomeration transition in a $\mathrm{PMMA} / \mathrm{SiO}_{2}$ hybrid suspension was considered based on the polymer solution theory. This sheds light on the limitations of the depletion aggregation theory, and a new agglomeration theory is proposed.

\section{EXPERIMENTAL PROCEDURE}

\section{Materials}

Silica nanoparticles. The colloidal $\mathrm{SiO}_{2}$ was prepared using the sol-gel method. The $\mathrm{SiO}_{2}$ nanoparticles were manufactured by Fuso Chemical Co., Ltd. (Osaka, Japan) (with unmodified or methyl-modified surfaces; an average diameter of 15 or $35 \mathrm{~nm}$ (spherical shape); and spherical, cocoon or aggregated shapes), and $12 \mathrm{wt} \% \mathrm{SiO}_{2}$ was dispersed in each unit of isopropyl alcohol (IPA). Data on the shapes, diameters and surface modifications of the $\mathrm{SiO}_{2}$ nanoparticles (Table 1 and Figure 1) were provided by Fuso Chemical Co., Ltd. The abbreviation '-non' in the table indicates that the surface of the colloidal nanosilica consists of silanol groups ( $\mathrm{SiOH})$, whereas '-methyl' indicates that the majority of the $\mathrm{SiOH}$ groups are chemically modified (replaced) with methyl groups.

Poly(methyl methacrylate). Commercial methyl methacrylate (manufactured by Wako Pure Chemical Industries, Ltd., Tokyo, Japan) was used as is, without any purification, for the PMMA. $\alpha, \alpha^{\prime}$-Azobisisobutyronitrile (manufactured by Kanto Chemical Co., Inc., Tokyo, Japan) was used as a radical initiator after recrystallization from methanol. Radical chain transfer agents were prepared in a variety of molecular weights to be used as the low-molecular-weight molecules. The molecular weight of the PMMA specimen prepared by radical polymerization was measured by a GPC (gel permeation chromatograph; HLC-8220GPC manufactured by Tosoh Corporation, Tokyo, Japan, with a TOSOHTSK gel GMHxL-G3000HxL-G2000HxL column system) equipped with a differential refractometer and using THF as an eluent. The molecular weight characteristics of the prepared PMMA were based on the standard monodisperse PMMA samples and are shown in Table 2.

The maximum molecular weight $\left(M W_{\max }\right)$ in the table represents the maximum molecular weight obtained in the GPC curve. All PMMA samples had broad molecular weight distributions in terms of the degree of heterogeneity (degree of dispersion), $M_{w} / M_{n}$. To clarify the dependence of the

Table 1 Characteristics of $\mathrm{SiO}_{2}$

\begin{tabular}{lll}
\hline & \multicolumn{2}{c}{ Particle size $(\mathrm{nm})$} \\
\cline { 2 - 3 } Shape & 15 & 35 \\
\hline Spherical & PL-2SL-non & PL-3L-methyl \\
Cocoon-shaped & PL-1SL-non & \\
Aggregated & PL-1SL-methyl & \\
& PL-1-non & \\
& PL-1-methyl &
\end{tabular}

Abbreviations: methyl, methyl modification; non, non-modification.



spherical



cocoon

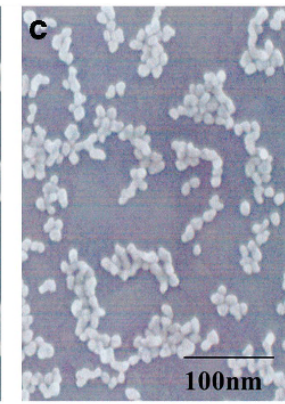

aggregated
Figure 1 TEM images of spherical, cocoon-shaped and aggregated $\mathrm{SiO}_{2}$ (a: spherical; b: cocoon; c: aggregated).

Table 2 Molecular weight characteristics of PMMA

\begin{tabular}{lccc}
\hline $\mathrm{M}_{\mathrm{n}} \times 10^{-4}$ & $\mathrm{M}_{\mathrm{w}} \times 10^{-4}$ & $\mathrm{MW}_{\max } \times 10^{-4}$ & $\mathrm{M}_{\mathrm{w}} / \mathrm{M}_{\mathrm{n}}$ \\
\hline 0.2 & 0.3 & 1.3 & 1.3 \\
0.4 & 0.7 & 10 & 2.1 \\
1.0 & 2.3 & 17 & 2.2 \\
5.0 & 9.6 & 120 & 1.9 \\
10 & 31 & 390 & 3.2 \\
\hline
\end{tabular}


dispersion-agglomeration transition behavior on the molecular weight of the polymer, it is fundamentally desirable to use a monodisperse sample with a narrow molecular weight distribution. However, more easily prepared polydisperse samples were used in our examination. The critical molecular weight $\left(M_{\mathrm{c}}\right)$ at which effective entanglement of the PMMA chains occurs has been reported to be approximately $3 \times 10^{4}$ based on the molecular weight dependence of the zero-shear melt viscosity. ${ }^{19}$ The red letters in the table indicate samples in which PMMA molecules with a MW of $M_{\mathrm{c}}$ or larger were included.

\section{Preparation of hybrid suspension}

The colloidal nanosilica was diluted to a specific concentration with THF purchased from Kanto Chemical, then stirred. To prepare suspensions with different PMMA concentrations for each molecular weight, a small amount of PMMA powder was added to the suspension and dissolved by stirring.

\section{Measurement}

Transmittance measurement. The dispersibility of the $\mathrm{SiO}_{2}$ was evaluated by measuring the transmittance of the suspension in a quartz cell with a light path length of $10 \mathrm{~mm}$ at room temperature using an ultraviolet-visible spectrophotometer (UV-visible, model V630, manufactured by JASCO Corporation, Tokyo, Japan).

Relative viscosity measurement. The relative viscosity was measured using an Ubbelohde improved capillary-type automatic kinematic viscosity tester (manufactured by Shibayama Scientific Co., Ltd, Tokyo, Japan). The viscosity meter was statically installed vertically in a precision constant-temperature water bath to allow the flow times of the various solutions between the gauge lines to be measured at a temperature of $25.00 \pm 0.01{ }^{\circ} \mathrm{C}$. The flow time of THF measured by the viscosity meter was $15.73 \mathrm{~s}$. The relative viscosity was calculated using the following equation, in which the solvent flow time is $t_{0}$, the polymer solution flow time is $t$ and the relative viscosity is $\eta_{\text {rel }}\left(t / t_{0}\right)$ :

$$
\text { Relative viscosity }\left(\eta_{\text {rel }}\right)=\frac{\text { polymer solution flow time }(t)}{\text { solvent flow time }\left(t_{0}\right)} \text {. }
$$

\section{RESULTS AND DISCUSSION}

\section{Agglomeration of silica nanoparticles and critical polymer} concentrations

The variation as a function of polymer concentration of the transmittance of the unmodified cocoon-shaped $\mathrm{SiO}_{2}$ nanoparticles (PL-1SL-non $\mathrm{SiO}_{2}, \varphi=15 \mathrm{~nm}$ ) diluted to $0.4 \mathrm{wt} \%$ with $\mathrm{THF}$ and added to PMMA $\left(M_{w}=9.6 \times 10^{4}, M_{w} / M_{n}=1.9\right)$ is shown in Figure 2. The distance between nanoparticles at the initial $\mathrm{SiO}_{2}$ concentration of $0.4 \mathrm{wt} \%$ was approximately $100 \mathrm{~nm}$, as derived by applying the model described above and proposed by Thuzyo and Harada, ${ }^{20}$ and a high transmittance was found for the entire wavelength range when the polymer concentration was within 0.0 $4.3 \mathrm{wt} \%$. When the polymer concentration increased from 5.3 to 7.3 wt $\%$, however, the transmittance decreased across the entire wavelength range. The reduction in transmittance was particularly significant in the low wavelength range, which is considered to be due to the agglomeration of the colloidal nanosilica, which is known to depend on the polymer concentration.

Figure 3 shows a plot of the transmittance in the $400-\mathrm{nm}$ range with respect to the polymer concentration from Figure 2. The transmittance of the PMMA/THF solution (two-component system, shown in red in the figure) did not depend on the polymer concentration and remained high even when the polymer concentration was high. In contrast, the transmittance dropped rapidly in the vicinity of $5 \mathrm{wt} \%$ PMMA for the three-component PL-1SL-non $\mathrm{SiO}_{2}$ $(\varphi=15 \mathrm{~nm}) /$ PMMA $\left(M_{w}=9.6 \times 10^{4}, M_{w} / M_{n}=1.9\right) /$ THF system, as shown in Figure 3. The PMMA concentration at which the transmittance drops rapidly is hereafter called the critical polymer concentration $\left(C^{\star}\right)$. No such $C^{\star}$ was observed in systems without $\mathrm{SiO}_{2}$, as described above. Furthermore, it was also found that when a white, turbid suspension at polymer concentrations of $C^{\star}$ or higher was left standing over a long period of time, sedimentation would occur, depending on the amount of agglomerated colloidal nanosilica.

However, the suspension became clear again when THF was used to redilute the solution to a polymer concentration of $C^{\star}$ or lower. However, when the PMMA concentration was increased, the $\mathrm{SiO}_{2}$



Figure 2 UV-visible spectra of a PMMA/PL-1-non $\mathrm{SiO}_{2}$ hybrid suspension in THF as a function of polymer concentration $\left(M_{W}=9.6 \times 10^{4}\right)$. 


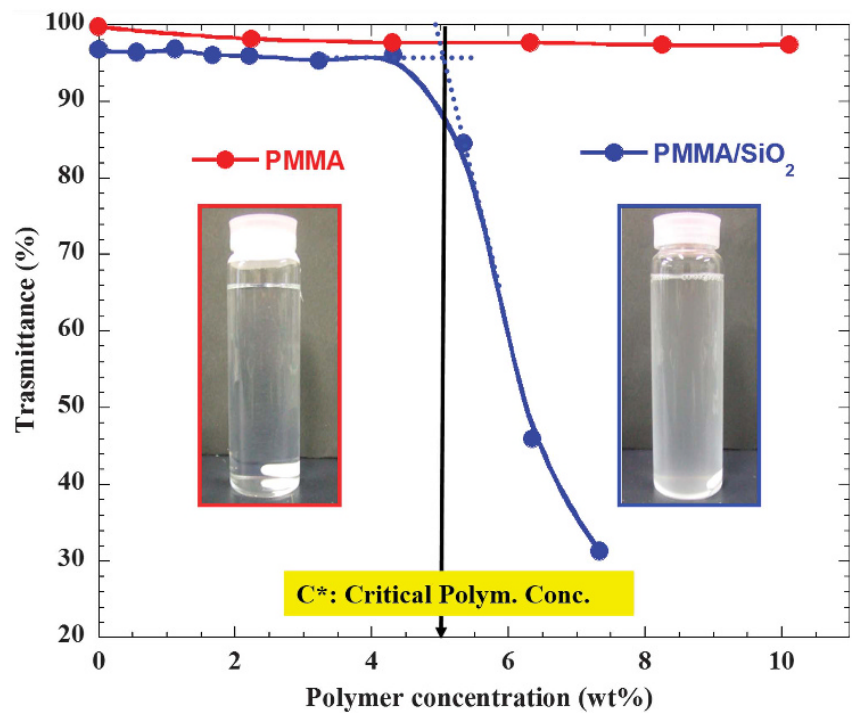

Figure 3 Comparison of the transmittance of $\mathrm{PMMA} / \mathrm{SiO}_{2}$ and $\mathrm{PMMA}$ in THF (wavelength: $400 \mathrm{~nm}$ ).

particles agglomerated because of the strong interactions between the polymers, even though the $\mathrm{SiO}_{2}$ nanoparticles were separated by approximately $100 \mathrm{~nm}$, causing the suspension to become cloudy once again. Therefore, $C^{\star}$ was considered to be equivalent to the concentration at which secondary agglomeration of the aggregated $\mathrm{SiO}_{2}$ nanoparticles occurred.

\section{Dependence of $C^{\star}$ on silica concentration}

To evaluate how $C^{\star}$ depends on the $\mathrm{SiO}_{2}$ concentration, an experiment was conducted in which PMMA was added to different initial $\mathrm{SiO}_{2}$ concentrations. First, cocoon-shaped PL-1SL-non $\mathrm{SiO}_{2}(\varphi=15$ $\mathrm{nm})$ was prepared at initial concentrations of $0.4,1.0$ and $2.0 \mathrm{wt} \%$, and then the polymer (PMMA, $M_{w}=9.6 \times 10^{4}, M_{w} / M_{n}=1.9$ ) was gradually added to produce a suspension. The change in the transmittance at $400 \mathrm{~nm}$ is shown in Figure 4.

Increasing the initial concentration of $\mathrm{SiO}_{2}$ when the polymer concentration was $0 \mathrm{wt} \%$ gradually reduced the transmittance, but in all cases, high levels of transmittance were sustained. A $C^{\star}$ value was observed for all suspensions in the vicinity of a $5 \mathrm{wt} \%$ polymer concentration, irrespective of the initial $\mathrm{SiO}_{2}$ concentration, as evident in Figure 4. Furthermore, the transmittance dropped more rapidly with higher initial $\mathrm{SiO}_{2}$ concentrations. The rate of the reduction in transmittance at polymer concentrations in excess of $C^{*}$ was equivalent to the speed at which the $\mathrm{SiO}_{2}$ particle aggregates were generated. This was attributed to the higher concentration of $\mathrm{SiO}_{2}$ in the suspension, which reduced the distance between the $\mathrm{SiO}_{2}$ particles, allowing them to interact and agglomerate. Furthermore, because $C^{\star}$ was observed to be independent of the $\mathrm{SiO}_{2}$ concentration under these conditions, it was concluded that the PMMA and $\mathrm{SiO}_{2}$ nanoparticles interacted with each other only weakly.

\section{Effects of $\mathrm{SiO}_{2}$ particle surface conditions and particle diameters on $C^{*}$}

The colloidal $\mathrm{SiO}_{2}$ was generated by a sol-gel reaction of tetraalkoxysilane and had an internal structure composed of siloxane groups (-Si-O-Si-). Furthermore, the surfaces of the unmodified particles were generally covered by silanol groups ( $\mathrm{Si}-\mathrm{OH})$, and the particles displayed a large specific surface area. The surface silanol groups had

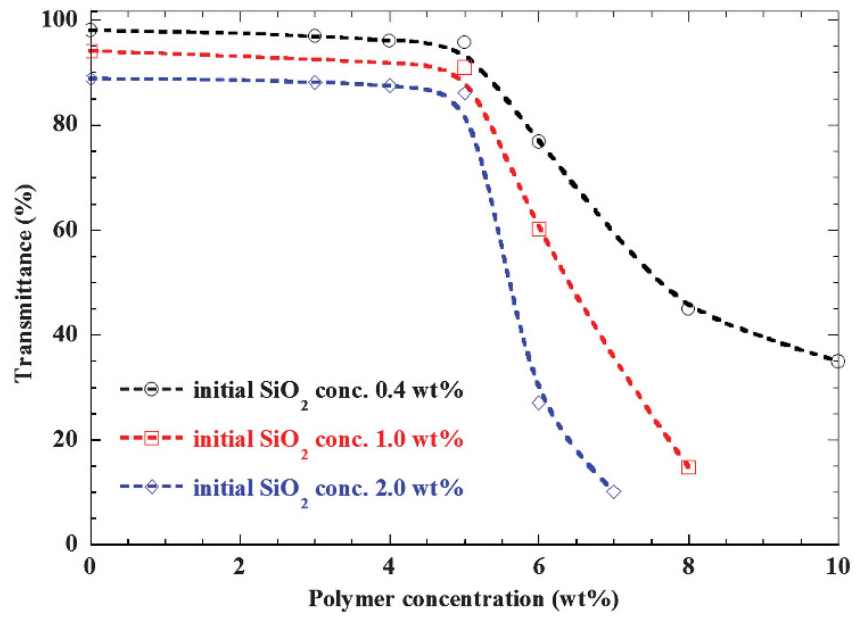

Figure 4 Polymer concentration dependence of the transmittance of a PMMA/PL-1SL-non $\mathrm{SiO}_{2}$ hybrid suspension in THF (wavelength: $400 \mathrm{~nm}$; $M_{W}$ of PMMA: $9.6 \times 10^{4}$ ).

the potential to interact with the hydroxyl groups of other $\mathrm{SiO}_{2}$ nanoparticles, the carbonyl groups of PMMA, or other species through hydrogen bonding. Such interactions are expected to significantly affect the physical properties of the dispersion, especially for initial particles with smaller diameters. However, Fourier-transform infrared spectroscopy showed no evidence of interaction between the hydroxyl groups of the $\mathrm{SiO}_{2}$ and the carbonyl groups of the PMMA in this experiment.

In the experiments in the presence of PMMA $\left(M_{w}=9.6 \times 10^{4}, M_{w} l\right.$ $M_{n}=1.9$ ) using different shapes of unmodified nanoparticles (average diameters of $15 \mathrm{~nm}$ ), such as cocoon (PL-1SL-non), spherical (PL2SL-non) and aggregated (P1-1-non) particles, $C^{\star}$ was $5 \mathrm{wt} \%$ in all cases, irrespective of the specific surface areas and numbers of silanol groups on the particle surfaces in the experiments. Thus, it was confirmed that for the evaluated experimental conditions, there was no dependence of $C^{\star}$ on the shape of the nanosilica particles, on the specific surface area of the particles or on the number of unmodified silanol groups.

In contrast, the modified nanosilica particles were generated by adding a small amount of methyl trialkoxysilane. These particles had a weaker ability to form hydrogen bonds because of their smaller numbers of silanol groups. Here, the effect of hydrogen bonding and of the steric repulsion of the methyl groups on $C^{\star}$ was examined using colloidal nanosilica with different surface conditions and particle diameters.

A plot of $C^{\star}$ versus the initial $\mathrm{SiO}_{2}$ concentration for various types of $\mathrm{SiO}_{2}$ nanoparticles with methyl-modified surfaces is shown in Figure 5. When the initial concentration of methyl-modified $\mathrm{SiO}_{2}$ nanoparticles was low, no clear $C^{\star}$ was observed, even when the polymer concentration was increased. However, a $C^{\star}$ gradually appeared when the initial $\mathrm{SiO}_{2}$ nanoparticle concentration was increased. The $\mathrm{C}^{\star}$ for the methyl-modified $\mathrm{SiO}_{2}$ nanoparticles was higher than that for the unmodified $\mathrm{SiO}_{2}$ and converged in the vicinity of 7-9 wt\%, when the initial concentration of the $\mathrm{SiO}_{2}$ nanoparticles was higher. This indicates that particle aggregates were less likely to form through hydrogen bonding between the $\mathrm{SiO}_{2}$ particle surfaces because there were fewer silanol groups on the surfaces than for the unmodified $\mathrm{SiO}_{2}$. Furthermore, this result is in contrast to the behavior for the unmodified $\mathrm{SiO}_{2}$ described above, in which $C^{\star}$ was in the vicinity of $5 \mathrm{wt} \%$ regardless of the shape of the 




Figure 5 Dependence of $\mathrm{C}^{*}$ on the initial concentrations of three types of methyl-modified $\mathrm{SiO}_{2}$.

$\mathrm{SiO}_{2}$ (spherical, cocoon or aggregated). For the methyl-modified $\mathrm{SiO}_{2}$, the $C^{*}$ was lower for the cocoon particles and for the aggregated particles (with initial particle diameters of $15 \mathrm{~nm}$ ) in comparison with that for the spherical particles (with an initial particle diameter of $35 \mathrm{~nm}$ ). This is believed to be due to the stronger interactions (hydrogen bonds) between particles with smaller diameters due to their larger relative surface areas. The polymer concentration that triggers agglomeration, therefore, was revealed to be dependent on the size of the initial particles and on the nature of the surface modification (that is, the number of silanol groups).

PMMA concentration and molecular weight dependence of $C^{\star}$ Figure 6 shows the dependence of $C^{\star}$ on the PMMA molecular weight for PL-1SL-non $\mathrm{SiO}_{2}(\varphi=15 \mathrm{~nm})$ at an initial concentration of 0.4 $\mathrm{wt} \%$. As clearly indicated in the figure, the transmittance rapidly decreased with increasing PMMA concentration, with $C^{\star}$ of $3.0 \mathrm{wt} \%$, $5.0 \mathrm{wt} \%$ and $9.0 \mathrm{wt} \%$ observed for PMMA samples with average molecular weights $\left(M_{w}\right)$ of $31 \times 10^{4}, 9.6 \times 10^{4}$ and $2.3 \times 10^{4}$ (most of which are above $M_{\mathrm{c}}$ ), respectively. Thus, $C^{\star}$ increased as the PMMA molecular weight decreased. The transmittance decreased slightly near polymer concentrations of approximately $20 \mathrm{wt} \%$ but remained constant thereafter for the PMMA sample with $M_{w}=0.7 \times 10^{4}$, which only contains a few molecular chains in excess of $M_{\mathrm{c}}$. Furthermore, the transmittance was nearly constant and remained high even at a polymer concentration of $50 \mathrm{wt} \%$ for the PMMA sample with an $M_{w}$ of $0.3 \times 10^{4}$, which does not contain any molecular chains in excess of $M_{\mathrm{c}}$. To interpret this peculiar molecular weight dependence of $C^{\star}$, we considered the molecular theory of polymer chains in polymer solutions.

A schematic diagram of the morphologies of the polymer (random-coil) chains with molecular weights that exceed $M_{\mathrm{c}}$ in solution is shown in Figure 7. This diagram shows the commonly accepted scheme for both ideal solutions using $\theta$ solvents and good real solutions using good solvents. ${ }^{21}$ Here, $C_{0}{ }^{*}$ represents the polymer concentration at which the polymer chains (random coils) in the solution come into contact with each other and start overlapping. In other words, at $C / C_{0}^{*}=1$, the ideal arrangement of the random coils is in a hexagonal close-packed structure. At $C / C_{0}{ }^{\star}<1$, the polymer

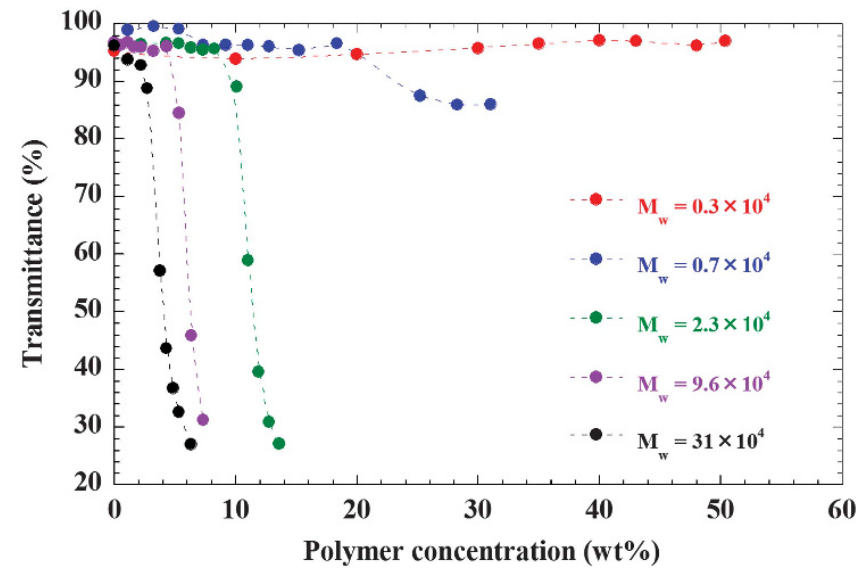

Figure 6 Polymer concentration and molecular weight dependence of the $\mathrm{C}^{*}$ of a PMMA/PL-1SL-non $\mathrm{SiO}_{2}$ hybrid suspension in THF (wavelength: $400 \mathrm{~nm}$; initial $\mathrm{SiO}_{2}$ conc.: 0.4 wt\%).



Figure 7 Model of the morphology of a polymer chain in solution in which the molecular weight of the polymer is above $M_{\mathrm{c}}$. A full color version of this figure is available at Polymer Journal online.

chains exist as mutually independent random-coil chains (isolated chains), and the molecular chains start to intrude on each other at $C /$ $C_{0}{ }^{*}>1$. Furthermore, overlapping of the molecular chains is known to occur above a certain molecular chain length (molecular weight: $M_{\mathrm{c}}$ ). Effective entanglement is indicated by $C / C_{0}{ }^{*} \gg 1$. The $M_{\mathrm{c}}$ of PMMA has been reported to be approximately $3 \times 10^{4}$, as described earlier. ${ }^{19}$ On the other hand, no effective entanglement appears with polymers with molecular weights lower than $M_{\mathcal{c}}$, even with $C / C_{0}{ }^{*}>1$ or even $C / C_{0}{ }^{*} \gg 1$.

The value of $C_{0}{ }^{*}\left(\mathrm{~g} \mathrm{~cm}^{-3}\right)$ is given by the following equation: ${ }^{21,22}$

$$
C_{0}^{*}=\frac{3 M}{4 \pi\left\langle S_{0}^{2}\right\rangle^{3 / 2} A_{v}}
$$

where $<S_{0}^{2}>$ represents the mean-square radius of gyration $\left(\AA^{2}\right), A_{\mathrm{v}}$ represents Avogadro's constant and $M$ represents the molecular weight of the polymer.

Although the $\left\langle S_{0}^{2}\right\rangle$ in the solution can be measured using the light scattering method, here, the value was estimated using the persistence length and the contour length, which are the parameters for the wormlike chain model used for polymer chains in ideal solutions $(\theta$ solvents $){ }^{23}$ The approximate calculation for the persistence length of the PMMA was conducted using the documented values for unperturbed coils $(8 \AA)$ obtained from neutron scattering data, ${ }^{24}$ while the contour length was derived from a comparison of the molecular weight $M$ of the polymer and the molecular weight $M_{L}$ per unit contour length $\left(M / M_{L}\right) .{ }^{25}$ The $C_{0}{ }^{*}$ values corresponding to the $M_{w}$ of the PMMA samples used in this experiment were 4.2 (0.042), 7.3 (0.073), $15(0.15), 28(0.28)$ and $43(0.43) \mathrm{g} \mathrm{dl}^{-1}\left(\mathrm{~g} \mathrm{~cm}^{-3}\right)$ for $M_{w}=31 \times 10^{4}, 9.6 \times 10^{4}, 2.3 \times 10^{4}$, 
$0.7 \times 10^{4}$ and $0.3 \times 10^{4}$, respectively. The value of $C_{0}{ }^{*}$ increased as the molecular weight decreased. The value of $C_{0}{ }^{*}$ in a good solvent is believed to be lower than that for an ideal chain because the solvent (THF) used for this $\mathrm{SiO}_{2}$ dispersion-agglomeration transition experiment was a good solvent for PMMA. ${ }^{26}$ Therefore, the spread in $\left\langle S_{0}{ }^{2}\right\rangle$ for real chains in a good solvent is more extensive than that for an ideal chain. ${ }^{27}$

However, as described above, the values of $C^{\star}$ for the PMMA used in this experiment, with $M_{w}$ values of $31 \times 10^{4}, 9.6 \times 10^{4}$ and $2.3 \times 10^{4}$, all of which contain components that exceed $M_{\mathrm{c}}$ (Figure 6), were 3.0, 5.0 and $9.0 \mathrm{wt} \%$, respectively. Therefore, the tendency of $C^{\star}$ to increase with decreasing molecular weight corresponded with the molecular weight dependence of $C_{0}^{*}$ from the polymer solution theory. However, no clear $C^{\star}$ was evident for the PMMA with $M_{w}=0.7 \times 10^{4}$, in which hardly any components exceeding $M_{\mathrm{c}}$ were present, or for the PMMA with $M_{w}=0.3 \times 10^{4}$, in which no such components were present. Furthermore, in the latter case, the transmittance did not decrease even for a polymer concentration of $50 \mathrm{wt} \%$, which exceeds the $C_{0}{ }^{*}$ value of $43 \mathrm{~g} \mathrm{dl}^{-1}$. Such results make it clear that when there are few interactions between the $\mathrm{SiO}_{2}$ nanoparticles and the PMMA molecular chains, the well-known 'depletion aggregation theory' for nanoparticles does not apply. Furthermore, the distance between nanoparticles was $\sim 100 \mathrm{~nm}$ when the initial silica concentration was $0.4 \mathrm{wt} \%$, and the driving force required to bring the nanoparticles to within the distances necessary for agglomeration cannot be explained by the depletion aggregation theory.

When the polymer concentration increases in solution, independent random coils start to come into contact with each other and to overlap, which finally leads to entanglement, as described earlier (Figure 7). The concentration at which random-coil chains come into contact with each other was estimated from the relative viscosity curve for the PMMA solution. Figure 8 shows the change in the relative viscosity with respect to the polymer concentration $C$ for the THF solution of PMMA $\left(M_{w}=9.6 \times 10^{4}\right)$ as well as for the THF-IPA solution. The dotted line corresponds to a relative viscosity $\left(\eta_{\text {rel }}\right)$ of 2 . The $C^{\star}$ values observed in this experiment with different types of $\mathrm{SiO}_{2}$

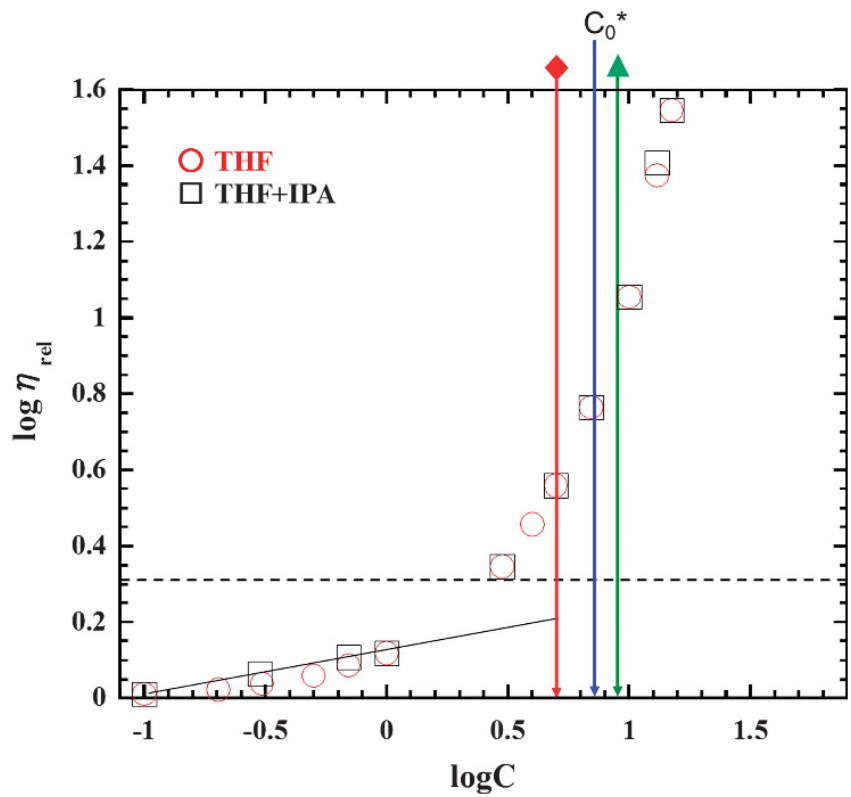

Figure 8 Polymer concentration dependence of the relative viscosities of PMMA/THF and PMMA/THF-IPA solutions. (The dotted line is the location of $\eta_{\text {rel }}=2$. The $C^{*}$ values of the data for the rhombus and triangle symbols are shown in Table 3.) are shown in Table 3. Because the relative viscosity of the solution started to increase at approximately $1 \mathrm{wt} \%$, we know that the PMMA chains started to come into contact with each other near this concentration, causing overlap and mutual intrusions of the polymers at concentrations above this point. Then, once entanglement occurred, the viscosity increased rapidly. The measured $C^{\star}$ values observed for the unmodified and methyl-modified $\mathrm{SiO}_{2}$ particles described above are summarized and shown in Figure 8, along with the estimated value of $C_{0}^{*}$ for an ideal solution. As evident in the figure, the $C^{\star}$ and $C_{0}{ }^{*}$ values were all $1 \mathrm{wt} \%$ or higher, at which point the PMMA molecular chains start to come into contact with each other, overlap, and become entangled. It is believed that once the PMMA chains with sufficient molecular weights (molecular chain lengths) to form entanglements come into contact, overlap, and become entangled, the viscosity of the suspension increases and the $\mathrm{SiO}_{2}$ particles dispersed in the polymer solution are cleared from the polymer domains. These domains become increasingly concentrated, forcing the $\mathrm{SiO}_{2}$ particles to approach each other and weakening the free Brownian motion, which results in a strong attraction between the $\mathrm{SiO}_{2}$ particles that results in agglomeration. The PMMA with a $M_{w}$ of $2.3 \times 10^{4}$, which contains components with $M W_{\max }$ of $17 \times 10^{4}$, above the $M_{\mathrm{c}}$, was considered to form many entanglements.

In the case of the PMMA sample with a $M_{w}$ of $0.3 \times 10^{4}$, which contained no components at or in excess of $M_{\mathcal{C}}$, however, it is believed that although the solution viscosity increased with increasing polymer concentration, no effective entanglement of the random-coil chains occurred, and as a result there was no driving force to bring the $\mathrm{SiO}_{2}$ particles closer together. Therefore, no reduction in the transmittance due to the agglomeration of the $\mathrm{SiO}_{2}$ nanoparticles was observed.

The 'dispersion-agglomeration transition' of the $\mathrm{SiO}_{2}$ particles in suspension strongly depended on the interactions between the PMMA random-coil chains that came into contact with each other, overlapped, and eventually became entangled. In other words, the contact, overlap and entanglement of the random-coil chains, which depended on the molecular weight and concentration of the PMMA, drove out the $\mathrm{SiO}_{2}$ particles and force them to approach each other. Thus, these interactions act as the principal driving force for the agglomeration of $\mathrm{SiO}_{2}$ particles. Furthermore, the $\mathrm{SiO}_{2}$ nanoparticles were stably dispersed in the PMMA with a molecular weight below $M_{\mathcal{C}}$, in which no effective entanglement occurs.

In the future, the dependence of $C^{\star}$ on PMMA molecular weight should be verified using PMMA samples with narrow molecular weight distributions, and the consistency with the solution theory of polymers must be investigated. These studies are currently ongoing in our laboratory.

Table $3 C^{*}$ values observed in this experiment with different types of $\mathrm{SiO}_{2}$.

\begin{tabular}{llc}
\hline & $C^{*}(\mathrm{wt} \%)$ & $\log C^{*}$ \\
\hline PMMMA/PL-2SL-non $\mathrm{SiO}_{2} / \mathrm{IPA}$ & 5.0 & 0.70 \\
aPMMA/PL-1SL-non $\mathrm{SiO}_{2} / \mathrm{IPA}$ & 5.0 & 0.70 \\
bPMMA/PL-1-non $\mathrm{SiO}_{2} / \mathrm{IPA}$ & 5.0 & 0.70 \\
cPMMA/PL-1-methyl $\mathrm{SiO}_{2} / \mathrm{IPA}$ & 7.0 & 0.85 \\
dPMMA/PL-3L-methyl $\mathrm{SiO}_{2} / \mathrm{IPA}$ & 7.5 & 0.88 \\
DPMMA/PL-1SL-methyl $\mathrm{SiO}_{2} / \mathrm{IPA}$ & 9.0 & 0.95
\end{tabular}

The $C^{*}$ values of all the samples in this study were between 5.0 and $9.0 \mathrm{wt} \%$. The $\mathrm{C}^{*}$ of a and $\mathrm{b}$ were the same as that of PMMA/PL-2SL-non $\mathrm{SiO}_{2} / \mathrm{IPA}(\downarrow)$. Because the values are close to $\mathrm{C}_{0}{ }^{*} 7.3 \mathrm{wt} \%\left(\log \mathrm{C}_{0}{ }^{*} 0.86\right), \mathrm{c}$ and $\mathrm{d}$ are not marked in Figure 8. 


\section{CONCLUSION}

When the polymer concentration reaches $C_{0}{ }^{*}$, at which point the random-coil chains of independent PMMA molecules in dilute solutions come into contact with each other and overlap, the domains of the entangled polymers begin to grow due to mutual intrusion between the independent random-coil chains. This drives the $\mathrm{SiO}_{2}$ nanoparticles in the PMMA/nanosilica suspensions out of the polymer domains, leading to rapid agglomeration of the nanoparticles in PMMA-THF solutions that contain polymer components with high molecular weights that equal or exceed $M_{\mathrm{c}}$. Thus, the molecular weight dependence of $C^{\star}$, the polymer concentration at which the $\mathrm{SiO}_{2}$ nanoparticles agglomerate and the transmittance of the suspension rapidly decreases, is closely related to the polymer entanglement that begins to occur at the concentration $C_{0}{ }^{*}$ derived from the diameter of the random-coil chains in suspension. For suspensions that do not include polymers (PMMA) of $M_{\mathrm{c}}$ or larger, however, there is no driving force for $\mathrm{SiO}_{2}$ particle agglomeration because no entanglement occurs between the random-coil polymer chains, and thus no clear $C^{\star}$ is observed. Thus, the criterion for entangling can be considered a dispersion stability condition. The dispersionagglomeration transition behavior observed in these experiments cannot be explained by the 'depletion aggregation theory' alone, suggesting that 'entangling agglomeration' driven by the entanglement of polymers is important.

Numerous fundamental studies on polymer/nanoparticle hybrid suspensions for such non-aqueous systems will be conducted in the future to contribute to the development of future technologies for creating hybrid materials.

\section{ACKNOWLEDGEMENTS}

The author would like to deeply thank Mr Masatoshi Sakai of Fuso Chemical Co., Ltd., who provided the colloidal silica (organosol) used in this study.

1 Krishnamoorti, R. \& Vaia, R. A. ACS Symposium Series 804 Polyme Nanocomposites-Synthesis, Characterization, and Modeling (American Chemical Society: Oxford University Press, 2001).

2 Wiley, K. \& Vaia, R. A. Polymer Nanocomposites (MRS Bulletin: Material Research Society, Pittsburgh, PA, 2007).

3 Vaia, R. A. \& Maguire, J. F. Polymer nanocomposites with prescribed morphology: going beyond nanoparticle-filled polymers. Chem. Mater. 19, 2736-2751 (2007).

4 Bhattachaya, S. N., Gupta, R. K. \& Kamal, R. M. Polymeric Nanoconposites (Hanser, Cincinanati, $\mathrm{OH}, 2008$ )

5 Jancar, J., Douglas, J. F., Starr, F. W., Kumar, S. K., Cassagnau, P., Lesser, A. J., Sternstein, S. S. \& Buehler, M. J. Current issues in research on structure-property relationships in polymer nanocomposites. Polymer. 51, 3321-3343 (2010).
6 Derjaguin, B. \& Landau, L. Theory of the stability of strongly charged lyophobic sols and of the adhesion of strongly charged particles in solutions of electrolytes. Prog. Surf. Sci. 43, 30-59 (1993).

7 Verwey, E. J. W. Theory of the interaction of two electrochemical double layers in combination with the forces between particles in suspensions and lyophobic sols. Chem. Weekbl. 39, 563-566 (1942).

8 Verwey, E. J. W. \& Overbeek, J. Th G. Theory of the Stability of Lyophobic Colloids (Elsevier, Amsterdam, 1948).

9 Meth, J. S., Zane, S. G., Chi, C., Londono, J. D., Wood, B. A., Cotts, P., Keating, M., Guise, W. \& Weigand, S. Development of filler structure in colloidal silica-polymer nanocomposites. Macromolecules 44, 8301-8313 (2011).

10 Asakura, S. \& Oosawa, F. On interaction between two bodies immersed in a solution of macromolecules. J. Chem. Phys. 22, 1255-1256 (1954).

11 Asakura, S. \& Oosawa, F. Interaction between particles suspended in solutions of macromolecules. J. Polym. Sci. 33, 183-192 (1958).

12 De Hek, H. \& Vrij, A. Phase separation in non-aqueous dispersions containing polyme molecules and colloidal spheres. J. Colloid Interface Sci. 70, 592-594 (1979).

13 De Hek, H. \& Vrij, A. Interractions in mixtures of colloidal silica spheres and polystyrene molecules in cyclohexane. J. Colloid Interface Sci. 84, 409-422 (1981).

14 Fleer, G. J., Scheutjens, J. H. M. H. \& Vincent, B. The stability of dispersions of hard spherical particles in the presence of nonadsorbing polymer. in 'Polymer Adsorption and Dispersion Stability ACS Symposium series' (eds Godhard, E. D. \& Vincent, B.) 245 (ACS, Washington, 1984).

15 Vincent, B., Edwards, J., Emmett, S. \& Jones, A. Depletion flocculation in dispersions of sterically stabilised particles ("SOFT SPHERES"). Colloids Surfaces 18, 261-281 (1986).

16 Sawaguchi, T., Ohaba, S., Sakurai, A., Hagiwara, T. \& Yano, S. Preparation of transparent PMMA/SiO2 nanohybrid: molecular weight dependence. Polymer Preprints, Japan 56, 762 (2007).

17 Sawaguchi, T. Preparation of transparent film of polymer/nano-particle hybrid Kobunshi High Polymers, Japan 56, 734 (2007).

18 Sawaguchi, T. A new method for preparation of nanoparticles-dispersing transparent polymeric materials based on a new dispersion-aggregation transition mechanism. Polymer Preprints, Japan 61, 4689-4690 (2012).

19 Ferry, J. D. Viscoelastic Properties of Polymers 377-378 (John Wiley \& Sons, New York, 1980).

20 Thuzyo, K. \& Harada, Y. On the distance between particles in synthetic polymer emulsion. Kolloid-Z. Z. Polym. 201, 66-68 (1965).

21 De Gennes, P. G. Scaling Concept in Polymer Physics 76-77 (Cornell University Press, Ithaca, New York, 1979)

22 Muroga, Y., Noda, I. \& Nagasawa, M. Investigation of local conformations of polyelectrolytes in aqueous solution by small-angle scatering: 1 . local conformations of poly(sodium acrylates). Macromolecules 18, 1576-1579 (1985).

23 Muroga, Y., Hayashi, K., Fukunaga, M., Kano, T., Shimizu, S. \& Kurita, K. "Change of the persistence lengths in the conformational transition pullulan- and amylasetribannilates". Biophys. Chem. 121, 96-104 (2006).

24 Kaji, K. Kobunshi no amorphous jotai. Zairyo Kagaku 25, 43-51 (1988).

25 Bnoit, H. \& Dory, P. Light scattering from non-Gaussian chains. J. Phys. Chem. 57, 958-963 (1953)

26 Katime, I., Cesteros, L. C. \& Ochoa, J. R. Viscometric behaviour of PMMA (3) /THF (1) Water (2) system. Polym. Bull. 6, 447-453 (1982).

27 Kato, T., Miyaso, K., Noda, I., Fujimoto, T. \& Nagasawa, M. Thermodynamics and hyrodynamic properties of linear polymer solutions. I. Light scattering monodisperse poly( $\alpha$-methylstyrene). Macomolecules 3, 777-786 (1970).

(c) (i) $\Theta$ This work is licensed under a Creative Commons Attribution-NonCommercial-NoDerivs 3.0 Unported License. To view a copy of this license, visit http://creativecommons. org/licenses/by-nc-nd/3.0/ 